\title{
Influencia de las redes de apoyo social en la evolución de la enfermedad de Alzheimer
}

\author{
Influence of social support networks on the \\ evolution of Alzheimer disease
}

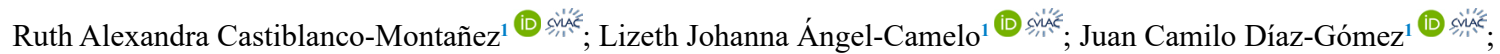

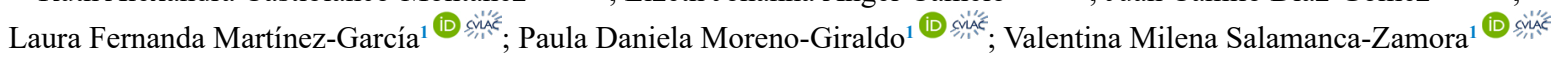

Forma de citar: Castiblanco-Montañez RA, Ángel-Camelo LJ, Díaz-Gómez JC, Martínez-García LF, Moreno-Giraldo PD, Salamanca-Zamora VM. Influencia de las redes de apoyo social en la evolución de la enfermedad de Alzherimer. Salud UIS. 53: e21019. doi: https://doi.org/10.18273/saluduis.53.e:21019 (c) (i)

\section{Resumen}

Introducción: el Alzheimer es la forma más común de demencia y una de las primeras causas de discapacidad. Objetivo: describir si en pacientes con Alzheimer el acompañamiento familiar, en comparación con el abandono, ralentiza la evolución de esta enfermedad. Metodología: revisión integrativa. Se seleccionaron artículos de los últimos cinco años, en idioma inglés, español y portugués, disponibles en las bases de datos PubMed, EBSCO HOST, Scielo, Clinicalkey, Google Académico; se utilizaron los términos MeSH y DeCS: Alzheimer, Continuity of Patient Care, family, Psychosocial Support Systems, junto con los operadores booleanos AND y OR. Se efectuó lectura crítica con las escalas STROBE y AMSTAR, y se clasificó el nivel de evidencia y grado de recomendación. Resultados: el diario vivir del cuidador evidencia sobrecarga física y psicológica que llevan a desistir del cuidado de los adultos que padecen Alzheimer. Conclusión: una red de apoyo estable garantiza mejores cuidados y educación que ralentizan la enfermedad.

Palabras clave: Enfermedad de Alzheimer; Continuidad de la Atención al Paciente; Apoyo Social; Sistemas de Apoyo Psicosocial; Atención de Enfermería; Familia.

\begin{abstract}
Introduction: Alzheimer is the most common form of dementia and one of the first causes of disability. Objective: To describe if in patients with Alzheimer's disease the family accompaniment, compared to the abandonment, slows the evolution of this disease. Methodology: Integrative review. Selected articles from the last five years, in English, Spanish and Portuguese, available in the PubMed, EBSCO HOST, Scielo, Clinicalkey, Google academic databases, using the terms MeSH and DeCS: Alzheimer, Continuity of Patient Care, family, Psychosocial Support Systems, along with Boolean operators "AND" and "OR". A critical reading was made with the STROBE and AMSTAR scales, and the level of evidence and degree of recommendation were classified. Results: The daily life of the caregiver shows physical and psychological overload that leads to giving up the care of adults suffering from Alzheimer. Conclusion: A stable support network ensures better care and education that slows the disease.
\end{abstract}

Keywords: Alzheimer Disease; Continuity of Patient Care; Social Support; Psychosocial Support Systems; Nursing Care; Family.

1. Fundación Universitaria de Ciencias de la Salud FUCS. Bogotá, Colombia.

Correspondencia: Dirección: Carrera 19 8a -32 Bogotá. Teléfono: +1 3538100 ext. 3604. Correo electrónico: racastiblanco@fucsalud.edu.co 


\section{Introducción}

La enfermedad de Alzheimer (EA) se manifiesta con la pérdida de la memoria, y en su etapa más grave, como un daño cerebral severo, que afecta funciones mentales superiores, principalmente la memoria, el pensamiento y el lenguaje ${ }^{1}$. Dentro de las enfermedades mentales, del sistema nervioso y de los trastornos seniles, la EA es una de las más importantes causas de discapacidad por el deterioro neuronal que, a su vez, afecta el proceso de sinapsis y degeneración neurofibrilar progresiva. Clínicamente se identifican cinco etapas: inicia con la fase preclínica; seguida del deterioro cognitivo leve; luego la presencia de una demencia leve, donde con mayor frecuencia se diagnostica la EA; después, se desarrolla la fase moderada por la afectación en las actividades diarias, y, por último, la etapa o fase avanzada, en donde existe una dependencia significativa o total, con pérdida de la capacidad de interacción social. La Organización Mundial de la Salud (OMS) asegura que la EA es la forma más común de demencia y representa entre un 60 y $70 \%$ de los casos ${ }^{2}$. Se calcula que entre un 5 a $8 \%$ de la población general de 60 años o más tiene la EA, lo que representaría 82 millones de casos para el 2030 y 152 millones para el $2050^{3}$.

Cuidar de un familiar dependiente genera cambios relevantes en los roles y las tareas, como la necesidad de asumir nuevas exigencias económicas y una reorganización en la vida familiar cotidiana. Cuando se diagnostica EA, las relaciones de la familia se ven modificadas. En la actualidad se ha demostrado que el acompañamiento familiar es un factor esencial en la evolución de esta patología, dado que el paciente cursa por diferentes etapas en las que debe asumir los cambios que trae esta condición; si las redes de apoyo del paciente no saben a lo que se enfrentan, puede aumentar la evolución de esta afectación neurológica ${ }^{4}$.

Para poder asumir y cumplir con la labor de ser un adecuado cuidador, se necesita que el profesional de enfermería brinde apoyo moral, asesore y ayude a suplir algunas necesidades, que proporcione información de los recursos que puedan facilitar el cuidado del paciente $^{5}$, ya que cuidar de un familiar con Alzheimer es complejo ${ }^{4}$.

Varios estudios reportan que el tratamiento para la EA se basa en evitar que el paciente se aparte y desarrolle depresión, ira, agresividad, delirios y trastornos del sueño que logran agudizar la enfermedad ${ }^{4}$. En las fases más avanzadas, el acompañamiento familiar prima para mantener la dimensión emocional en adecuadas condiciones y la racionalidad del afectado como una constante conexión de emociones y sentimientos. Uno de los grandes ejemplos es el amor, pues permite que el paciente reconozca estas emociones y las convierta en sensaciones positivas que traigan consigo recuerdos que logren rememorar sensaciones y situaciones vividas ${ }^{5}$.

La atención a pacientes con EA requiere un trato multidisciplinar, en el cual deben estar al tanto profesionales y familiares. Los cuidados de enfermería están encaminados a realizar las actividades básicas de cuidado del paciente y prevención de problemas asociados a la movilidad, y están también encaminadas al cuidador ${ }^{6}$.

El propósito de estas acciones es generar conciencia y aportar excelentes esfuerzos. Con ello se brinda dignidad y bienestar a las personas que se enfrentan a las enfermedades neurocognitivas y también a sus familias para que presten la asistencia requerida con el afecto que se merecen ${ }^{7}$. Por esta razón, algunas entidades prestadoras de servicios de salud implementan programas de apoyo y capacitación a cuidadores primarios de pacientes con Alzheimer, dirigidos por profesionales de la salud, a través de los cuales la familia del paciente con la EA obtiene recursos para evitar el avance de la misma ${ }^{7}$, puesto que le permite conocer sobre la enfermedad y así orientar los cuidados terapéuticos de forma más efectiva buscando que a nivel social se reflexione sobre la importancia de la prevención de la $\mathrm{EA}^{8}$.

En este sentido, este estudio busca describir si en pacientes con Alzheimer avanzado el acompañamiento familiar durante este proceso, en comparación con el abandono, ralentiza la evolución de esta enfermedad.

\section{Metodología}

Revisión integrativa de la literatura ${ }^{9}$. En la primera etapa se planteó el problema y la pregunta con la estrategia PICO. P: paciente con Alzheimer avanzado; $\mathrm{I}$ : acompañamiento familiar; $\mathrm{C}$ : abandono, y $\mathrm{O}$ : ralentización de la enfermedad. En la segunda etapa se establecieron como criterios de inclusión: artículos publicados entre los años 2014 y 2019, lo cual incluye tesis; capítulos de libros en inglés, español y portugués, de cualquier nacionalidad y diseño; textos completos que cumplieran con el objetivo del estudio. Fueron excluidos los artículos duplicados y que no respondieran al tema de interés. 
La búsqueda sistemática de documentos, como tercera etapa, se efectuó en las bases de datos PubMed, EBSCO HOST, Scielo, Clinicalkey, y Google académico; a través de las ecuaciones construidas a partir de los DeCS y MeSH Alzheimer AND Continuity of Patient Care, Alzheimer AND family, Alzheimer OR descuidado y Alzheimer and Psychosocial Support Systems y los operadores booleanos AND y OR.

En el proceso de selección de unidades de análisis se construyó una matriz organizada en columnas de Microsoft Office Excel $^{\circledR}$ donde se organizaron artículos de acuerdo con la base de datos, según idioma, ecuación, resultados, título, resumen, artículos definitivos, duplicados, referencia, diseño, objetivo, año de publicación, nivel de evidencia y grado de recomendación, de forma que al finalizar la búsqueda se obtuvieron los artículos que más se relacionaran directamente con la investigación.

El proceso de selección de unidades de análisis encontradas por los criterios de exclusión e inclusión se realizó según título, objetivo, diseño, resumen y año de cada artículo, por lo que al finalizar la búsqueda se obtuvieron los artículos que se relacionaron directamente con la investigación secundaria, y se construyó una matriz organizada en columnas de Excel.

Las unidades documentales fueron sometidas a lectura crítica para determinar la validez de los resultados por medio de escalas; para estudios observacionales se aplicó la escala STROBE, según los parámetros establecidos como criterio de inclusión: un puntaje superior a 13 puntos; para estudios de revisión sistemática se usó la escala AMSTAR, que sigue los parámetros como criterio de inclusión de un puntaje superior a 6 puntos para la aceptación del artículo. Las unidades de análisis finales se clasificaron por nivel de evidencia y grado de recomendación a partir de la propuesta del Centre for Evidence Based Medicine (CEMB) de Oxford ${ }^{10}$. De acuerdo con las consideraciones éticas vigentes para Colombia, este estudio se clasifica sin riesgo ético por ser de tipo documental.

\section{Resultados}

La búsqueda arrojó un total de 161805 artículos, divididos de la siguiente manera: PubMed 2,8\%, EBSCO HOST 1,7\%, Scielo 0,3\%, Clinicalkey 9,7\%, Google Académico 85\%; 243 por título, 106 por resumen, donde 58 de ellos cumplían con los criterios de inclusión y exclusión. Treinta unidades de análisis $(51,7 \%)$ fueron publicadas en inglés, entre el año 2015 y 2019 (véase Gráfica 1).

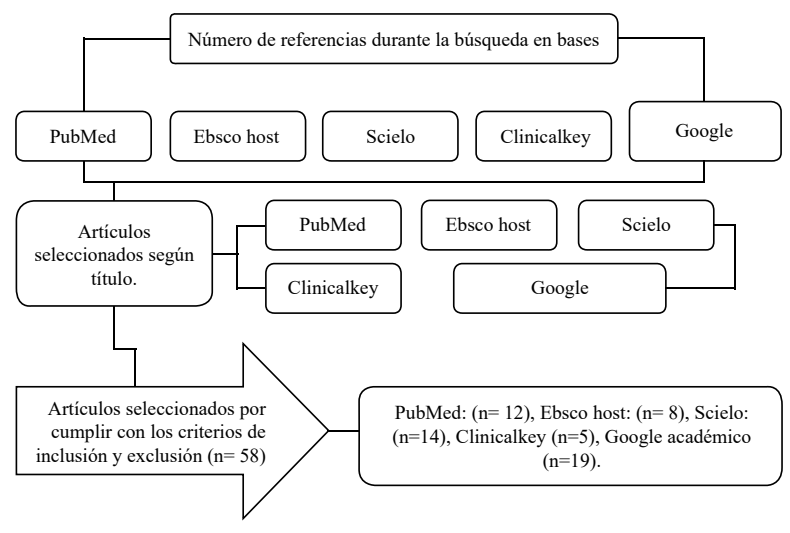

Gráfica 1. Proceso de búsqueda y selección de unidades de análisis.

Fuente. Los autores, 2021.

Tras el análisis de los datos surgieron cuatro temas dominantes: razones del abandono familiar en pacientes con Alzheimer, implicaciones del acompañamiento familiar o el abandono para el desarrollo de la enfermedad, fases del Alzheimer donde hay más incidencia de abandono y qué hace el enfermero para fortalecer el acompañamiento.

\section{Razones del abandono familiar en pacientes con Alzheimer}

Es complejo garantizar una atención óptima, ya que los cuidadores asumen grandes cargas de trabajo tanto objetivas como subjetivas, en referencia con el tiempo empleado para cuidar al enfermo y al cumplimiento de lo que esta persona necesita. Según la gravedad de la enfermedad, se hizo referencia a la percepción negativa que tiene quien realiza la tarea y los sentimientos que esta situación le generón ${ }^{11}$. Se evidenció que las razones de abandono familiar hacia los pacientes con Alzheimer prevalecen en un $45 \%$ por la sobrecarga que presenta el cuidador $^{12}$. Cuidar a personas con Alzheimer genera un desgaste tanto físico como psicológico en los cuidadores como consecuencia de la dedicación, pues repercute en su vida social, personal y económica; esto se conoce como síndrome del cuidador o sobrecarga del cuidador ${ }^{13}$.

En un $34 \%$ se evidenció abandono por la sintomatología que experimenta el cuidador, entre estos: miedo, estrés, falta de tiempo y sentimiento de soledad. Esto impacta en la vida de los cuidadores familiares, al generar angustia emocional, sufrimiento, tristeza, agotamiento, y al afectar directamente la calidad de vida y la salud mental. Asimismo, se pueden desarrollar situaciones que afectan el bienestar del cuidador, entre estas, reducción de las horas de sueño y descanso, agotamiento, cansancio y fatiga; las cuales generan sobrecarga y déficit del cuidado ${ }^{14}$. 
Cuidar a una persona con EA no solo genera un costo emocional, sino que también requiere tiempo y dinero por los servicios, generalmente, no remunerados; en ocasiones, no todos los cuidadores de personas con EA tienen la comodidad de asistir a sus familiares 24 horas y cumplir con las responsabilidades económicas, por lo cual dejan de lado sus trabajos y familias, generando cambios drásticos como no compartir el mismo tiempo ${ }^{15}$.

El 11,4\% reflejó abandono con la progresividad de la enfermedad, ya que demanda mayor disposición y asistencia del cuidador; las responsabilidades no cambian o aumentan de manera inmediata, pero poco a poco se vuelven más marcadas a medida que disminuyen las habilidades de las personas con Alzheimer. En 8,5\% se evidenció el abandono por parte de sus familiares, debido principalmente a la falta de conocimiento acerca de la enfermedad y a la complejidad para comunicarse con su familiar. A medida que la enfermedad avanza, la familia experimenta situaciones muy difíciles, ya que además de la aparición de la discapacidad, se pierde toda relación compartida entre el enfermo y su familia; estos cambios exigen modificaciones en las funciones y los roles que los miembros del grupo familiar desempeñan. Algunas personas sienten temor de no poder brindar los cuidados necesarios a sus familiares, ya que consideran no tener los conocimientos necesarios para asistirlos, así que prefieren delegar esta función a otras personas como enfermeras o cuidadores encargados de centros para adultos mayores ${ }^{16}$.

\section{Implicaciones del acompañamiento fami- liar o el abandono para el desarrollo de la enfermedad}

El acompañamiento familiar demostró tener una implicación del $56 \%$ en la prevención del deterioro tanto cognitivo como físico en las personas con Alzheimer, lo cual evidencia que el sistema de apoyo como red fundamental permite una mayor satisfacción en el cuidado, ralentizando así el desarrollo de la enfermedad. El 31\% de las familias relacionaron su asistencia a pacientes con EA al parentesco y a la preocupación de que no fueran bien cuidados por personas particulares; esto permitió que el deterioro fuera más lento, ya que la díada generó una mayor comunicación y empatía frente a los síntomas.

En cuanto al abandono, se demostró en un 12,5\% el aumento de la sintomatología por la pérdida de atención familiar y la deserción del cuidador frente a las etapas que esté cursando el enfermo, aumentando así el deterioro cognitivo por la inactividad frecuente en actividades sociales que puede brindar el cuidado familiar o cuidador principal ${ }^{17}$.

Existen distintas implicaciones frente al acompañamiento familiar de una persona con Alzheimer avanzado. En primer lugar, se afirmó que los pacientes con enfermedades neurológicas que viven solos o con un solo cuidador necesitan un sistema de apoyo para cuidar sus síntomas psicológicos, sociales y culturales; aquellas preocupaciones, esperanzas, afectos y sentimientos presentados por las personas con EA con un acompañante permanente durante el proceso de la enfermedad reducen la sintomatología, aumentando así sentimientos de empatía y altruismo que permiten un sentido de alegría y ternura. Por lo tanto, inducen al crecimiento de la autoestima y favorecen la percepción de ser significativo y útil ${ }^{18}$.

La acción cuidadora cambia con el tiempo según evoluciona la enfermedad. Al avanzar el estadio de la enfermedad, aumenta el grado de implicación del cuidador en las actividades básicas de la vida diaria, lo cual implica la toma de decisiones y valoración de la evolución cognitiva $\mathrm{u}$ otros aspectos fundamentales como el control de los síntomas o las alteraciones conductuales. En sí, el acompañamiento puede ralentizar el deterioro cognitivo y retrasar el avance de la enfermedad de Alzheimer; si los pacientes participan con frecuencia en actividades sociales, su deterioro empezará a ser más lento ${ }^{19}$.

Se considera que la EA es una enfermedad familiar, pues para afrontar esta situación el cuidador necesita restablecer sus papeles personales, sociales y de trabajo, por lo cual modifica su entorno personal, laboral y psicológico, ya que debe acomodar su vida en pro del afectado. El compromiso de la familia y los cuidadores durante el proceso desempeña un papel fundamental en la rehabilitación y en la lenta progresividad de la enfermedad ${ }^{20,21}$.

Frente a esto, cada investigación reveló que el abandono durante el desarrollo de la enfermedad aumenta la sintomatología por la pérdida de atención familiar por parte del cuidador, aumentando así el deterioro cognitivo a causa de la disminución de las actividades sociales. El desconocimiento de los cuidados necesarios para aquellos pacientes aumenta el abandono, ya que los cuidadores informales no saben cómo sobrellevar los cambios que trae consigo la enfermedad, tanto para ellos como para el paciente, por ejemplo, el cambio en sus papeles diarios. Estos cambios generan afectaciones 
socioeconómicas, emocionales, aumento de la carga física y conflictos familiares, de acuerdo con los cambios de comportamiento del enfermo, ya que es un impacto muy negativo en la vida de los cuidadores, lo que los hace más susceptibles a cuadros de depresión y ansiedad ${ }^{22-24}$.

\section{Fases del Alzheimer donde hay más inci- dencia de abandono}

La enfermedad de Alzheimer tiende a desarrollarse y empeorar de forma gradual a lo largo de los años. Con el tiempo, el paciente va cursando diferentes etapas que generan sobre el cuidador un desgaste físico, emocional y mental, lo que trae como consecuencia deserción a la hora del cuidado.

La enfermedad cuenta con tres etapas principales: temprana o leve, media o moderada y avanzada o final. En la primera se cuenta con la independencia del paciente, pero con pérdida de la memoria lápsica, como nombres cotidianos o lugares de los objetos. La etapa moderada suele ser la más prolongada, donde la persona necesita cada día más colaboración para realizar tareas diarias; esta etapa afecta las funciones mentales, específicamente la esfera emocional y el pensamiento. Por último, en la fase avanzada ya no hay control ni conexión con el entorno, subdividiendo el desarrollo de síntomas y complicaciones del Alzheimer y terminando con la dependencia total, por lo general, a cargo del cuidador primario ${ }^{25-27}$.

Estas etapas se caracterizan por pérdida de la memoria, dificultad para resolver las tareas, cambios de personalidad, agresividad, dificultad para expresarse, ubicarse y alimentarse, lo que lleva consigo a que el cuidador maneje todos estos cambios y, si no cuenta con una red de apoyo, se hace más difícil la comprensión de los síntomas y, por lo tanto, su cuidado ${ }^{27}$.

En la etapa avanzada se desarrollan múltiples factores para el abandono del familiar. Uno de los principales es el incremento de gastos para el cuidado de una persona con dependencia total. Aparte, y no menos importante, la relación entre cuidador y paciente empieza a verse sustancialmente comprometida al ver que su existencia se borra rápidamente en su familiar, pues ya no reconoce quién es y, por tanto, se vive la pérdida de su familiar aun estando en vida, conocida también como la muerte social. La única salida de los familiares es encontrar apoyo en el personal de la salud, lo cual se considera como abandono por no poder brindar el cuidado constante que se debe dar ${ }^{28-32}$.
Las revisiones de las unidades finales de análisis afirmaron que la fase de Alzheimer avanzado presenta mayor incidencia del abandono familiar (80\%) por el incremento de dependencia de los enfermos, lo que significa para sus cuidadores mayor dificultad en la asistencia.

\section{Qué hace el enfermero para fortalecer el acompañamiento}

Se evidenciaron 44 respuestas acerca de las intervenciones primarias de enfermería en pacientes con EA, las cuales equivalen al $76 \%$. Los artículos equivalentes a este porcentaje llegaron a la conclusión de que el profesional desempeña un papel fundamental durante el apoyo en un $64 \% \%^{33}$.

Para llevar esto a cabo, los familiares deben recibir las pautas necesarias acerca del manejo de la enfermedad, como son la educación y los planteamientos de intervenciones individuales; de esta forma, cuentan con una red de apoyo para generar una satisfacción plena y, a la vez, determinan un cuidado holístico, minimizando así la sobrecarga del cuidador ${ }^{34-36}$.

El $35 \%$ de los resultados refirieron al personal de la salud como la fuente de atención primaria que brinda cuidados, lo cual da mayor tranquilidad, ya sea de forma domiciliaria o institucional, para comprender las etapas del Alzheimer. Se crea así un mayor manejo de este, dado que la enfermería no se enfatiza solo en el paciente sino también en la familia, formando así terapias activas tales como centros de apoyo a cuidadores que permitan interactuar y compartir experiencias con otras personas que se encuentren pasando por las mismas circunstancias, junto con terapias psicoemocionales que permitan al cuidador aumentar su conocimiento y disminuir el nivel de estrés que lleva a la sobrecarga y al abandono $0^{37,38}$.

La educación juega un papel fundamental a la hora de querer disminuir el estrés en el cuidador, ya que, según la revisión de los artículos, la gran mayoría arrojaron que las faltas de conocimientos no permiten que el cuidador primario logre desenvolverse con mayor tranquilidad en el transcurso de la enfermedad. Una persona que conoce la patología y sabe acerca del deterioro neurológico está más dispuesta a colaborar en actividades que ralenticen la enfermedad. Por esto, la enfermería no debe abandonar la parte de educación, sino más bien fortalecerla, ya que cuando el familiar 
adquiere conocimientos acerca de esta, se muestra más dispuesto a colaborar, debido a que se siente apoyado por el grupo de salud $\mathrm{d}^{39,40}$.

Es importante recordar que se debe organizar una red de apoyo de atención integral conformada por enfermería, medicina, psicología y trabajo social para brindar al cuidador un apoyo incondicional que disminuya las cargas y muestre alternativas durante el avance de la enfermedad ${ }^{41,42}$.

Para finalizar, el $1 \%$ de los artículos revisados no dio respuesta a la pregunta susceptible. Sin embargo, si la dan a otras preguntas.

\section{Discusión}

Las bases de datos consultadas reflejan la efectividad de la red de soporte social en la disminución del proceso degenerativo. Kaplan define esta red en términos de lazos perdurables que desempeñan un papel importante en la integración psicológica y física de una persona ${ }^{43}$. En este sentido, se ha descrito la díada paciente-cuidador o red de apoyo. En esta se generan implicaciones en el vínculo como consumo de tiempo, que traen consigo impacto en varios ámbitos como: actitudinal, familiar, cultural, espiritual y económico, y afectan la forma y la calidad de vida de ambas partes, creando la necesidad de un cuidado conjunto o, como lo nombran algunos autores, "el binomio" 4 . En este sentido, la familia es la principal fuente de apoyo para el afrontamiento de la condición, pues mejora la calidad de vida del paciente en el curso de la enfermedad ${ }^{45-47}$.

Cabe aclarar que el profesional de enfermería puede ser parte de esa red de apoyo a través de estrategias de comunicación y acompañamiento, que respondan a las necesidades encontradas frente al paciente, tales como demostrar que no están solos, la escucha activa o compañía en las oraciones. Por otro lado, las alternativas que se plantean, cuando no es posible un contacto físico, es que la enfermería potencie el uso de herramientas como el internet, las llamadas y demás TIC (correo electrónico, redes sociales), con el fin de disminuir la incertidumbre, la ansiedad, el aislamiento y la depresión ${ }^{46,48,49}$.

Al reconocer que los cuidadores requieren asesoría y acompañamiento en los momentos de cuidado, se deben realizar intervenciones individuales y grupales donde la enfermería brinde apoyo psicosocial implementando conocimientos y autonomía en la díada cuidadorpaciente. En relación con el fortalecimiento de este vínculo, se ha centrado la educación en los potenciales y responsabilidad del paciente y la importancia del cuidador principal en el acompañamiento y apoyo psicosocial para favorecer el afrontamiento y adaptación a las situaciones irreversibles que los pacientes vivencian en torno a su patología. Asimismo, la implementación de programas dirigidos al cuidador aporta en la adquisición de destrezas y habilidades, reduce la sobrecarga, mejora la funcionalidad familiar, permite el establecimiento de metas de cuidado y resalta la labor del cuidador como una acción significativa en la ralentización de la condición ${ }^{50-53}$.

\section{Conclusiones y recomendaciones}

Se afirma que en pacientes con Alzheimer avanzado el acompañamiento familiar ralentiza la evolución de esta enfermedad siendo este un factor fundamental, de modo que el paciente que no cuenta con una red de apoyo es susceptible a involucionar prontamente. A partir de las diferentes fases por las cuales transcurre un paciente con EA, se observa que en las etapas finales existe mayor dependencia, menor disponibilidad de tiempo para las diferentes tareas y la red de apoyo desiste del cuidado, demandando esta actividad a otra persona. El profesional de enfermería debe identificar las necesidades que aquejan al cuidador para así fortalecer y dar una introducción de la patología, aclarando la importancia del acompañamiento familiar, los factores positivos al implementar cuidados basados en la evidencia, para así retrasar la evolución de la patología, mantener el estado físico y emocional, que cubra las necesidades del paciente, logrando un equilibro en el desarrollo de la enfermedad para contrarrestar la sobrecarga del cuidador.

\section{Referencias}

1. Larramendi Esandi N, Canga-Armayor A. Familia cuidadora y enfermedad de Alzheimer: una revisión bibliográfica. Gerokomos. 2011; 22 (2).

2. Donoso A. La enfermedad de alzheimer. Rev Chil Neuro-psiquiatr. 2016; 41(2). doi: http://dx.doi. org/10.4067/S0717-92272003041200003

3. Demencia. Organización mundial de la salud. 2017 https://www.who.int/es/news-room/fact-sheets/ detail/dementia

4. Henley D, Sundell K, Sethuraman G y Schneider L. Adverse events and dropouts in Alzheimer's disease studies: What can we learn? Alzheimers Dement. 2015; 11(1): 24-31. doi: 10.1016/j.jalz.2013.11.008

5. Ibiza D. Alzheimer, un reto para la familia. 2015. https://www.diariodeibiza.es/vida-y-estilo/ salud/2015/09/21/alzheimer-reto-familia/794495.html 
6. Martínez D, Rosales A, Martínez C, Socrates J. Differential effects of a psycho-educational intervention on caregivers of people with Alzheimer's acute disease. Alzheimer's \& Dementia. 2017; 13(7): 503-504. doi: 10.1016/j. jalz.2017.06.565

7. Medina J. Aplicativo par a el apoyo del cuidador familiar de pacientes con Alzheimer. Neurología. 2016. Tesis. http://polux.unipiloto.edu. co:8080/00003533.pdf

8. Alzheimer: la importancia de la detección temprana. 2017. https://tn.com.ar/salud/noticias/2017/09/21/ alzheimer-la-importancia-de-la-detecciontemprana/

9. Sasso Mendes K, Campos Pereira Silveira R, Galvão C. Revisão integrativa: método de pesquisa para a incorporação de evidências na saúde e na enfermagem integrative literature review: a research method to incorporate evidence in health care and nursing. 2008; 17(4). 758-764.

10. Centre for Evidence-based Medicine (CEBM)Levels of Evidence. 2011; http://www.cebm. net/mod_product/design/files/CEBM-Levels-ofEvidence-2.1.pdf

11. Sinha P, Desai NG, Prakash O, Kushwaha S, Tripathi CB. Caregiver burden in Alzheimer-type dementia and psychosis: A comparative study from India. Asian J Psychiatr. 2017; 26: 86-91. doi: https://doi. org/10.1016/j.ajp.2017.01.002

12. Antelo P, Espinosa P. La influencia del apoyo social en cuidadores de personas con deterioro cognitivo o demencia. Revista de Estudios e Investigación en Psicología y Educación. 2017; 14. doi: https://doi. org/10.17979/reipe.2017.0.14.2235

13. Römhild J, Fleischer S, Meyer G, Stephan A, Zwakhalen S, Leino-Kilpi $\mathrm{H}$, et al. Inter-rater agreement of the Quality of Life-Alzheimer's Disease (QoL-AD) self-rating and proxy rating scale: secondary analysis of RightTimePlaceCare data. Health Qual Life Outcomes. 2018; 16(1). doi: 10.1186 / s12955-018-0959

14. Navarro Martínez M, Jiménez Navascués L, García Manzanares C, Perosanz Calleja M, Blanco Tobar E. Alzheimer's patients and their caregivers: Nursing interventions. Gerokomos. 2018; 29(2): 79-82.

15. Hsin Hsiao Y, Chang Chih H, Gean P. Impact of social relationships on Alzheimer's memory impairment: mechanistic studies. J Biomedical Science. 2018; 25(3). doi: 10.1186/s12929-018-0404-X

16. Rodríguez P, Álvarez G, Martínez B, Cañizares S, Borroto C, Haraicy P. El síndrome del cuidador en cuidadores principales de ancianos con demencia Alzhéimer. Gaceta Médica Espirituana. 2017; 19(1).
17. Ohta Y, Yamashita T, Hishikawa N, Sato K, Hatanaka $\mathrm{N}$, Takemoto M, et al. Affective improvement of neurological disease patients and caregivers using an automated telephone call service. J Clin Neurosci. 2018; 56: 74-78. doi: https://doi.org/10.1016/j. ajp.2017.01.002

18. Swall A, Ebbeskog B, Lundh Hagelin C, Fagerberg I. Stepping out of the shadows of Alzheimer's disease: a phenomenological hermeneutic study of older people with Alzheimer's disease caring for a therapy dog. Int J Qual Stud Health Well-being. 2017; 12(1). doi: 10.1080/17482631.2017.1347013

19. Asís C, Costa R. Calidad de vida de los ancianos con Alzheimer. DSpace RIUFF. 2017. Tesis. https:// app.uff.br/riuff/bitstream/1/5930/1/Disserta\%C3\% A7\%C3\%A3oDefesaCintiaRAQUEL.pdf

20. Marinês L, Pizolotto A, Hildebrandt L, Cocco M, Gindri D. Organização da família no cuidado ao idoso com doença de Alzheimer. Espaço para Saúde. 2015; 16(4).

21. Edneia P. Reabilitação cognitiva em grupo com de foco em metas funcionais para pacientes com doença de Alzheimer leve: um ensaio clínico controlado. San Pablo. 2016. doi: 10.11606/D.5.2016.tde06122016-160738

22. Campos M, Amorim F, Forlenza O. Social skills and well-being among family caregivers to patients with Alzheimer's disease. Rev. 2016. doi: 10.1590/010160830000000143

23. Leiva V, Hernández ME, Aguirre E. Experiencias de familias que conviven con una persona con diagnóstico de Alzheimer. Rev Enfermería Act Costa Rica. 2016; 30: 1-22. doi: http://dx.doi. org/10.15517/revenf.v0i30.22550

24. Caldas C, Costa M, Chave M, Cruz T. Cognitive stimulation for older people with Alzheimer's disease performed by the caregiver. Rev Bras Enferm. 2015; 68(3): 510-516. doi: https://doi. org/10.1590/0034-7167.2015680319i

25. Kimura NRS, Baptista MAT, Santos RL, Portugal MDG, Johannenssen A, Barca ML, et al. Caregivers' perspectives of quality of life of people with young- and late-onset Alzheimer disease. J Geriatr Psychiatry Neurol. 2018; 31(2): 76-83. doi: 10.1177 / 0891988718759601

26. Ilha S; Stein D; Backes S; Porto D; Tarouco B; Teda M. Doença de alzheimer na pessoa idosa/ família: Dificuldades vivenciadas e estratégias de cuidado. Rev Escola Anna Nery. 2015; 20(1). doi: 10.5935/1414-8145.20160019

27. Esandi N, Canga A. Enfoque sistémico familiar: un marco para la atención profesional en la enfermedad de Alzheimer. Gerokomos. 2016; 27(1). 
28. Onieva-Zafra MD1, Hernández-García L, GonzalezDel-Valle MT, Parra-Fernández ML, FernandezMartinez E. Music intervention with reminiscence therapy and reality orientation for elderly people with Alzheimer disease living in a nursing home: A Pilot Study. Holist Nurs Pract. 2018; 32(1): 43-50. doi: 10.1097/ HNP.0000000000000247

29. Cerquera Córdoba A, Pabón Poches D, Granados Latorre F, Galvis Aparicio M. Burden in caregivers of Alzheimer's disease patients, and its relation with their incomes. Psicogente. 2016; 19(36): 240-251. doi: http://dx.doi.org/10.17081/psico.19.36.1295

30. Hansel C, Marins A, Silva J. Mudanças de comportamento em idosos com Doença de Alzheimer e sobrecarga para o cuidador. Rev Escola Anna Nery. 2015; 20(2). doi: 10.5935/14148145.20160048

31. Manzini C, Brigola A, Pavarinia S, Carvalho F. Fatores associados à resiliência de cuidador familiar de pessoa com demência: revisão sistemática. Rev Escola Anna Nery. 2016.

32. Baldin L, Quintino D, Silva N, Kusumota L, Marques S. Sintomas neuropsiquiátricos do idoso com doença de Alzheimer e o desgaste do cuidador familiar. Rev Latinoamericana Enferm. 2016; 24.

33. Mendes M, Filgueira C, Santos A. The care in Alzheimer's disease: social representations of family caregivers. Saúde e Sociedade. 201; 25(1).

34. Monteiro D, Sales Dos Santos I, Ianne R, Nascimento M, Dos Santos Silva M. Os Cuidadores de idosos com doença de Alzheimer E A Enfermagem: Uma Revisão sistemática de literatura. Cieh. 2017. doi: https://doi.org/10.1590/S0104-12902015142591

35. Duan Y, Liming L, Chen J, Wu C, Liang J, Zheng $\mathrm{Y}$, et al. Psychosocial interventions for Alzheimer's disease cognitive symptoms: a Bayesian network meta-analysis. BMC Geriatrics. 2018; 18(1). doi: https://doi.org/10.1186/s12877-018-0864-6

36. Zubatsky M, Aragon Prada M, Fátima M, Rainey P, Martin R. Navigating without a roadmap: Challenges of early Alzheimer's caregivers with their health care team. Glob Qual Nurs Res. 2016. doi: $10.1177 / 2333393616673465$

37. Czekanski K. The experience of transitioning to a caregiving role for a family member with Alzheimer's disease or related dementia. Am J Nursing. 2017; 117(9): 24-32.

38. Lanzonia A, Fabbob A, Bassoc D, Pedrazzinid $\mathrm{P}$, Bortolomiole $\mathrm{E}$, Jonesf $\mathrm{M}$, et al. Interventions aimed to increase independence and well-being in patients with Alzheimer's disease: Review of some interventions in the Italian context. Neurol Psych Brain. 2018; 30: 137-143. doi: https://doi. org/10.1016/j.npbr.2018.10.002
39. Silva L, Camacho A, Lopes F, Gurgel J, Lima T, Queiroz R. Vulnerability of caregivers of the elderly with dementia. Rev Bras Enferm. 2017; 70(4).

40. Díaz A, Farías I, Márquez S. Estudio sobre la convivencia familiar cuando un integrante tiene Alzheimer a partir del cuidador principal. Desarr Eco Sociedad. 2017; 6(1): 185-203. doi: https://doi. org/10.38017/issn.2322-804

41. Pascual Y, Garzón M, Ravelo M. Relación entre dependencia en pacientes con enfermedad de Alzheimer y sobrecarga en el cuidador principal. Rev Cubana Enfermería. 2018; 34(1).

42. Dura E, Garces J. La teoría del apoyo social y sus implicaciones para el ajuste psicosocial de los enfermos oncológicos. Rev Psicol Social. 2015; $34(1)$.

43. Sánchez L, Carrillo G. Competencia para el cuidado en el hogar diada persona con cáncer en quimioterapia-cuidador familiar. Psicooncología. 2017;14(1).

44. Vega O, González D. Apoyo social: elemento clave en el afrontamiento de la enfermedad crónica. Enferm Global. 2019; 16.

45. Fernández A, Manrique F. Rol de la enfermería en el apoyo social del adulto mayor. Enferm Global. 2016; 19.

46. Torres X, Carreño S. Factores que influencian la habilidad y sobrecarga del cuidador familiar del enfermo crónico. Rev Univ Ind Santander Salud 2017; 49(2). doi: http://dx.doi.org/10.18273/revsal. v49n2-2017006

47. Galván G. La enfermera en la red social de apoyo. Rev Enferm Inst Mex Seguro Soc. 2019; 17(2): 61-62.

48. Rodríguez L, Chaparro L. Soporte social y sobrecarga en cuidadores: revisión integrativa. Rev Cuidarte. 2020; 11(1).

49. Herrera A, Flórez I, Romero E. Soporte social a cuidadores familiares de personas con enfermedad crónica en Cartagena. Aquichan. 2017; 12(3).

50. Hernández N, Moreno C. Necesidades de cuidado de la díada cuidador-persona: expectativa de cambio en intervenciones de enfermería. Rev Cuidarte. 2016; 5(2): 748-756.

51. Ferrer M, Cibanal L. El aprendizaje de cuidados familiares a pacientes con Alzheimer. Revisión bibliográfica. Rev Cult Cuidados. 2016; 5(2): 748-756.

52. Ruiz E, Poyo A. Revisión de una Intervención de Enfermería. NIC: 007040 Apoyo al Cuidador Principal. Rev Enferm CyL. 2016; 8(2).

53. Venegas M. Atención de enfermería al familiar cuidador primario del paciente oncológico. U Javeriana. 2018; Tesis. https://www.javeriana.edu. co/biblos/tesis/enfermeria/tesis56.pdf 